Proyecciones Journal of Mathematics

Vol. 31, No 3, pp. 235-246, September 2012.

Universidad Católica del Norte

Antofagasta - Chile

\title{
On $(i, j)-\omega$-preopen sets
}

\author{
N. RAJESH \\ Rajah Serfoji Govt. College, India \\ and \\ JAMAL M. MUSTAFA \\ Al al-Bayt University, Jordan \\ Received: August 2010. Accepted : May 2012
}

\begin{abstract}
In this paper, we introduce and study the notion of $(i, j)-\omega$-preopen sets as a generalization of $(i, j)$-preopen sets in bitopological space.
\end{abstract}

Keywords : Bitopological spaces, $(i, j)$-preopen sets, $(i, j)$-precontinuous functions.

Subjclass : [2000], 54C10, 54D10. 


\section{Introduction and Preliminaries}

Generalized open sets play a very important role in General Topology and they are now the research topics of many topologists worldwide. Indeed a significant theme in General Topology and Real analysis concerns the variously modified forms of continuity, separation axioms etc. by utilizing generalized open sets. The concept of a bitopological space was introduced by Kelly [4]. On the other hand Jelic [2] introduced the concept of preopen sets in bitopological spaces. In this paper, we introduce and study the notion of $(i, j)-\omega$-preopen sets as a generalization of $(i, j)$-preopen sets in bitopological spaces. Throughout this paper, spaces means bitopological spaces on which no separation axioms are assumed unless otherwise mentioned. For a subset $A$ of $X$, the closure and the interior of $A$ are denoted by $(A)$ and $(A)$, respectively. A subset $A$ of a bitopological space $\left(X, \tau_{1}, \tau_{2}\right)$ is said to be $(i, j)$-preopen [2] if $A \subset \tau_{i^{-}}\left(\tau_{j^{-}}(A)\right)$, where $i, j=1,2$ and $i \neq j$. The complement of an $(i, j)$-preopen set is said to be $(i, j)$-preclosed set ([3], [5]). The $(i, j)$-preclosure [5] of $A$, denoted by $(i, j)-p(A)$, is defined by the intersection of all $(i, j)$-preclosed sets containing $A$. The $(i, j)$-preinterior [6] of $A$, denoted by $(i, j)-p(A)$, is defined by the union of all $(i, j)$-preopen sets contained in $A$. A function $f:\left(X, \tau_{1}, \tau_{2}\right) \rightarrow\left(Y, \sigma_{1}, \sigma_{2}\right)$ is said to be $(i, j)$-precontinuous $([3],[5])$ if the inverse image of every $\sigma_{i}$-open set in $\left(Y, \sigma_{1}, \sigma_{2}\right)$ is $(i, j)$-preopen in $\left(X, \tau_{1}, \tau_{2}\right)$, where $i \neq j, i, j=1,2$.

\section{2. $(i, j)-\omega$-preopen sets}

Definition 2.1. A subset $A$ is said to be $(i, j)-\omega$-preopen if for each $x \in$ $A$ there exists an $(i, j)$-preopen set $U_{x}$ containing $x$ such that $U_{x} \backslash A$ is a countable set. The complement of an $(i, j)-\omega$-preopen subset is said to be $(i, j)-\omega$-preclosed.

The family of all $(i, j)-\omega$-preopen (resp. $(i, j)-\omega$-preclosed) subsets of a space $\left(X, \tau_{1}, \tau_{2}\right)$ is denoted by $(i, j)-\omega P O(X)$ (resp. $\left.(i, j)-\omega P C(X)\right)$. Also, The family of all $(i, j)$ - $\omega$-preopen sets of $\left(X, \tau_{1}, \tau_{2}\right)$ containing $x$ is denoted by $(i, j)-\omega P O(X, x)$.

It is clear that every $(i, j)$-preopen set is $(i, j)-\omega$-preopen. The following example shows that the converse is not true in general.

Example 2.2. Let $X=\{a, b, c\}, \tau_{1}=\{,\{a\}, X\}$ and $\tau_{2}=\{,\{b\}, X\}$. Then $\{a, c\}$ is $(i, j)$ - $\omega$-preopen but not $(i, j)$-preopen in $\left(X, \tau_{1}, \tau_{2}\right)$. 
Lemma 2.3. $A$ subset $A$ of a bitopological space $\left(X, \tau_{1}, \tau_{2}\right)$ is $(i, j)-\omega$ preopen if and only if for every $x \in A$, there exists an $(i, j)$-preopen subset $U_{x}$ containing $x$ and a countable subset $C$ such that $U_{x} \backslash C \subset A$.

Proof. Let $A$ be $(i, j)$ - $\omega$-preopen and $x \in A$, then there exists an $(i, j)$ $\omega$-preopen subset $U_{x}$ containing $x$ such that $U_{x} \backslash A$ is countable. Let $C=$ $U_{x} \backslash A=U_{x} \cap(X \backslash A)$. Then $U_{x} \backslash C \subset A$. Conversely, let $x \in A$. Then there exists an $(i, j)-\omega$-preopen subset $U_{x}$ containing $x$ and a countable subset $C$ such that $U_{x} \backslash C \subset A$. Thus, $U_{x} \backslash A \subset C$ and $U_{x} \backslash A$ is countable.

Theorem 2.4. Let $\left(X, \tau_{1}, \tau_{2}\right)$ be a bitopological space and $C \subset X$. If $C$ is $(i, j)$ - $\omega$-preclosed, then $C \subset K \cup B$ for some $(i, j)-\omega$-preclosed subset $K$ and a countable subset $B$.

Proof. If $C$ is $(i, j)$ - $\omega$-preclosed, then $X \backslash C$ is $(i, j)$ - $\omega$-preopen and hence for every $x \in X \backslash C$, there exists an $(i, j)$ - $\omega$-preopen set $U$ containing $x$ and a countable set $B$ such that $U \backslash B \subset X \backslash C$. Thus $C \subset X \backslash(U \backslash B)=$ $X \backslash(U \cap(X \backslash B))=(X \backslash U) \cup B$. Let $K=X \backslash U$. Then $K$ is $(i, j)$ - $\omega$-preclosed such that $C \subset K \cup B$.

Proposition 2.5. The union of any family of $(i, j)$ - $\omega$-preopen sets is $(i, j)$ $\omega$-preopen.

Proof. If $\left\{A_{\alpha}: \alpha \in \Lambda\right\}$ is a collection of $(i, j)-\omega$-preopen subsets of $X$, then for every $x \in \bigcup_{\alpha \in \Lambda} A_{\alpha}, x \in A_{\gamma}$ for some $\gamma \in \Lambda$. Hence there exists an $(i, j)$-preopen subset $U$ of $X$ containing $x$ such that $U \backslash A_{\gamma}$ is countable. Now as $U \backslash \bigcup_{\alpha \in \Lambda} A_{\alpha} \subset U \backslash A_{\gamma}$ and thus $U \backslash \bigcup_{\alpha \in \Lambda} A_{\alpha}$ is countable. Therefore, $\bigcup_{\alpha \in \Lambda} A_{\alpha}$ is $(i, j)$ - $\omega$-preopen.

Definition 2.6. The union of all $(i, j)$ - $\omega$-preopen sets contained in $A \subset X$ is called the $(i, j)-\omega$-preinterior of $A$, and is denoted by $(i, j)-\omega p(A)$. The intersection of all $(i, j)-\omega$-preclosed sets of $X$ containing $A$ is called the $(i, j)-\omega$-preclosure of $A$, and is denoted by $(i, j)-\omega p(A)$.

Theorem 2.7. Let $A$ and $B$ be subsets of $\left(X, \tau_{1}, \tau_{2}\right)$. Then the following properties hold:

(i) $(i, j)-\omega p(A)$ is the largest $(i, j)$ - $\omega$-preopen subset of $X$ contained in A. 
(ii) $A$ is $(i, j)$ - $\omega$-preopen if and only if $A=(i, j)-\omega p(A)$.

(iii) $(i, j)-\omega p((i, j)-\omega p(A))=(i, j)-\omega p(A)$.

(iv) If $A \subset B$, then $(i, j)-\omega p(A) \subset(i, j)-\omega p(B)$.

(v) $(i, j)-\omega p(A \cap B) \subset(i, j)-\omega p(A) \cap \subset(i, j)-\omega p(B)$.

(vi) $(i, j)-\omega p(A) \cup(i, j)-\omega p(B) \subset(i, j)-\omega p(A \cup B)$.

Proof. (v). Since $A \cap B \subset A$ and $A \cap B \subset B$, by (iv), we have $(i, j)$ $\omega p(A \cap B) \subset(i, j)-\omega p(A)$ and $(i, j)-\omega p(A \cap B) \subset(i, j)-\omega p(B)$. Therefore, $(i, j)-\omega p(A \cap B) \subset(i, j)-\omega p(A) \cap(i, j)-\omega p(B)$.

(vi). We have $(i, j)-\omega p(A) \subset(i, j)-\omega p(A \cup B)$ and $(i, j)-\omega p(B) \subset(i, j)$ $\omega p(A \cup B)$. Then we obtain $(i, j)-\omega p(A) \cup(i, j)-\omega p(B) \subset(i, j)-\omega p(A \cup B)$. The other proof are obvious.

Theorem 2.8. Let $A$ and $B$ be subsets of $\left(X, \tau_{1}, \tau_{2}\right)$. Then the following properties hold:

(i) $(i, j)-\omega p(A)$ is the smallest $(i, j)$ - $\omega$-preclosed subset of $X$ containing A.

(ii) $A$ is $(i, j)-\omega$-preclosed if and only if $A=(i, j)-\omega p(A)$.

(iii) $(i, j)-\omega p((i, j)-\omega p(A))=(i, j)-\omega p(A)$.

(iv) If $A \subset B$, then $(i, j)-\omega p(A) \subset(i, j)-\omega p(B)$.

(v) $(i, j)-\omega p(A) \cup(i, j)-\omega p(B) \subset(i, j)-\omega p(A \cup B)$.

(vi) $(i, j)-\omega p(A \cap B) \subset(i, j)-\omega p(A) \cap(i, j)-\omega p(B)$.

Proof. The proofs follows from the definitions.

Theorem 2.9. Let $\left(X, \tau_{1}, \tau_{2}\right)$ be a bitopological space and $A \subset X$. A point $x \in(i, j)-\omega p(A)$ if and only if $U \cap A \neq \emptyset$ for every $U \in(i, j)-\omega P O(X, x)$. 
Proof. Suppose that $x \in(i, j)-\omega p(A)$. We shall show that $U \cap A \neq \emptyset$ for every $U \in(i, j)-\omega P O(X, x)$. Suppose that there exists $U \in(i, j)$ $\omega P O(X, x)$ such that $U \cap A=\emptyset$. Then $A \subset X \backslash U$ and $X \backslash U$ is $(i, j)-\omega$ preclosed. Since $A \subset X \backslash U,(i, j)-\omega p(A) \subset(i, j)-\omega p(X \backslash U)$. Since $x \in(i, j)$ $\omega p(A)$, we have $x \in(i, j)-\omega p(X \backslash U)$. Since $X \backslash U$ is $(i, j)-\omega$-preclosed, we have $x \in X \backslash U$; hence $x \notin U$, which contradicts the fact that $x \in U$. Therefore, $U \cap A \neq \emptyset$. Conversely, suppose that $U \cap A \neq \emptyset$ for every $U \in(i, j)-\omega P O(X, x)$. We shall show that $x \in(i, j)-\omega p(A)$. Suppose that $x \notin(i, j)-\omega p(A)$. Let $U=X \backslash(i, j)-\omega p(A)$, then $U \in(i, j)-\omega P O(X, x)$ such that $U \cap A=(X \backslash(i, j)-\omega p(A)) \cap A \subset(X \backslash A) \cap A=\emptyset$. This is a contradicition to $U \cap A \neq \emptyset$; hence $x \in(i, j)-\omega p(A)$.

Theorem 2.10. Let $\left(X, \tau_{1}, \tau_{2}\right)$ be a bitopological space and $A \subset X$. Then the following propeties hold:

(i) $(i, j)-\omega p(X \backslash A)=X \backslash(i, j)-\omega p(A)$;

(i) $(i, j)-\omega p(X \backslash A)=X \backslash(i, j)-\omega p(A)$.

Proof. (i). Let $x \in X \backslash(i, j)-\omega p(A)$. Since $x \notin(i, j)-\omega p(A)$, there exists $V \in(i, j)-\omega P O(X, x)$ such that $V \cap A=\emptyset$; hence we obtain $x \in(i, j)$ $\omega p(X \backslash A)$. This shows that $X \backslash(i, j)-\omega p(A) \subset(i, j)-\omega p(X \backslash A)$. Let $x \in$ $(i, j)-\omega p(X \backslash A)$. Since $(i, j)-\omega p(X \backslash A) \cap A=\emptyset$, we obtain $x \notin(i, j)-\omega p(A)$; hence $x \in X \backslash(i, j)-\omega p(A)$. Therefore, we obtain $(i, j)-\omega p(X \backslash A)=X \backslash(i, j)$ $\omega p(A)$.

(ii). Follows from (i).

Definition 2.11. A subset $B_{x}$ of a bitopological space $\left(X, \tau_{1}, \tau_{2}\right)$ is said to be an $(i, j)$ - $\omega$-preneighbourhood of a point $x \in X$ if there exists an $(i, j)$ - $\omega$-preopen set $U$ such that $x \in U \subset B_{x}$.

Theorem 2.12. A subset of a bitopological space $\left(X, \tau_{1}, \tau_{2}\right)$ is $(i, j)-\omega$ preopen if and only if it is an $(i, j)-\omega$-preneighbourhood of each of its points.

Proof. Let $G$ be an $(i, j)-\omega$-preopen set of $X$. Then by definition, it is clear that $G$ is an $(i, j)-\omega$-preneighbourhood of each of its points, since for every $x \in G, x \in G \subset G$ and $G$ is $(i, j)$ - $\omega$-preopen. Conversely, suppose $G$ is an $(i, j)$ - $\omega$-preneighbourhood of each of its points. Then for each $x \in G$, there exists $S_{x} \in(i, j)-\omega P O(X)$ such that $S_{x} \subset G$. Then $G=\bigcup\left\{S_{x}: x \in G\right\}$. Since each $S_{x}$ is $(i, j)$ - $\omega$-preopen, $G$ is $(i, j)-\omega$-preopen in $\left(X, \tau_{1}, \tau_{2}\right)$. 
Theorem 2.13. If each nonempty $(i, j)$-preopen set of a bitopological space $\left(X, \tau_{1}, \tau_{2}\right)$ is uncountable, then $(i, j)-p(A)=(i, j)-\omega p(A)$ for each subset $A \in \tau_{1} \cap \tau_{2}$.

Proof. Clearly $(i, j)-\omega p(A) \subset(i, j)-p(A)$. On the other hand, let $x \in$ $(i, j)-p(A)$ and $B$ be an $(i, j)-\omega$-preopen subset containing $x$. Then by Lemma 2.3, there exists an $(i, j)$-preopen set $V$ containing $x$ and a countable set $C$ such that $V \backslash C \subset B$. Thus $(V \backslash C) \cap A \subset B \cap A$ and so $(V \cap A) \backslash C \subset$ $B \cap A$. Since $x \in V$ and $x \in(i, j)-p(A), V \cap A \neq \emptyset$ and $V \cap A$ is $(i, j)$-preopen since $V$ is $(i, j)$-preopen and $A \in \tau_{1} \cap \tau_{2}$. By the hypothesis each nonempty $(i, j)$-preopen set of $X$ is uncountable and so is $(V \cap A) \backslash C$. Thus $B \cap A$ is uncountable. Therefore, $B \cap A \neq \emptyset$ which means that $x \in(i, j)-\omega p(A)$.

Corollary 2.14. If each nonempty $(i, j)$-preclosed set of a bitopological space $\left(X, \tau_{1}, \tau_{2}\right)$ is uncountable, then $(i, j)-p(A)=(i, j)-\omega p(A)$ for each $A \in \tau_{1} \cap \tau_{2}$.

Theorem 2.15. If every $(i, j)$-preopen subset of $X$ is $\tau_{i}$-open in $\left(X, \tau_{1}, \tau_{2}\right)$, then $(X,(i, j)-\omega P O(X))$ is a topological space.

Proof. (i). We have $\emptyset, X \in(i, j)-\omega P O(X)$. (ii). Let $U, V \in(i, j)$ $\omega P O(X)$ and $x \in U \cap V$. Then there exist $(i, j)$-preopen sets $G, H \in X$ containing $x$ such that $G \backslash U$ and $H \backslash V$ are countable. And $(G \cap H) \backslash(U \cap$ $V)=(G \cap H) \cap((X \backslash U) \cup(X \backslash V)) \subset(G \cap(X \backslash U)) \cup(H \cap(X \backslash V))$. Hence $(G \cap H) \backslash(U \cap V)$ is countable and by hypothesis, the intersection of two $(i, j)$-preopen sets is $(i, j)$-preopen. Hence $U \cap V \in(i, j)-\omega P O(X)$. (iii). Let $\left\{U_{i}: i \in I\right\}$ be any family of $(i, j)-\omega$-preopen sets of $X$. Then, by Proposition $2.5 \bigcup_{i=1}^{n} U_{i}$ is $(i, j)$ - $\omega$-preopen.

\section{3. $(i, j)-\omega$-precontinuous functions}

Definition 3.1. A function $f:\left(X, \tau_{1}, \tau_{2}\right) \rightarrow\left(Y, \sigma_{1}, \sigma_{2}\right)$ is said to be $(i, j)$ $\omega$-precontinuous if the inverse image of every $\sigma_{i}$-open set of $Y$ is $(i, j)-\omega$ preopen in $X$, where $i \neq j, i, j=1,2$.

It is clear that every $(i, j)$-precontinuous function is $(i, j)-\omega$-precontinuous but not conversely.

Example 3.2. Let $X=\{a, b, c\}, \tau=\{,\{a\}, X\}$ and $\sigma=\{,\{a, c\}, X\}$. Clearly the identity function $f:(X, \tau) \rightarrow(X, \sigma)$ is $(i, j)$ - $\omega$-precontinuous but not $(i, j)$-precontinuous. 
Theorem 3.3. For a function $f:\left(X, \tau_{1}, \tau_{2}\right) \rightarrow\left(Y, \sigma_{1}, \sigma_{2}\right)$, the following statements are equivalent:

(i) $f$ is $(i, j)-\omega$-precontinuous;

(ii) For each point $x$ in $X$ and each $\sigma_{i}$-open set $F$ in $Y$ such that $f(x) \in F$, there is an $(i, j)-\omega$-preopen set $A$ in $X$ such that $x \in A, f(A) \subset F$;

(iii) The inverse image of each $\sigma_{i}$-closed set in $Y$ is $(i, j)-\omega$-preclosed in $X$;

(iv) For each subset $A$ of $X, f((i, j)-\omega p(A)) \subset \sigma_{i}-(f(A))$;

(v) For each subset $B$ of $Y,(i, j)-\omega p\left(f^{-1}(B)\right) \subset f^{-1}\left(\sigma_{i^{-}}(B)\right)$;

(vi) For each subset $C$ of $Y, f^{-1}\left(\sigma_{i^{-}}(C)\right) \subset(i, j)-\omega p\left(f^{-1}(C)\right)$.

Proof. $\quad(\mathrm{i}) \Rightarrow$ (ii): Let $x \in X$ and $F$ be a $\sigma_{i}$-open set of $Y$ containing $f(x)$. By (i), $f^{-1}(F)$ is $(i, j)$ - $\omega$-preopen in $X$. Let $A=f^{-1}(F)$. Then $x \in A$ and $f(A) \subset F$.

(ii) $\Rightarrow\left(\right.$ i): Let $F$ be $\sigma_{i}$-open in $Y$ and let $x \in f^{-1}(F)$. Then $f(x) \in F$. By (ii), there is an $(i, j)-\omega$-preopen set $U_{x}$ in $X$ such that $x \in U_{x}$ and $f\left(U_{x}\right) \subset F$. Then $x \in U_{x} \subset f^{-1}(F)$. Hence $f^{-1}(F)$ is $(i, j)$ - $\omega$-preopen in $X$.

(i) $\Leftrightarrow\left(\right.$ iii): This follows due to the fact that for any subset $B$ of $Y, f^{-1}(Y \backslash B)$ $=X \backslash f^{-1}(B)$.

(iii) $\Rightarrow$ (iv): Let $A$ be a subset of $X$. Since $A \subset f^{-1}(f(A))$ we have $A \subset$ $f^{-1}\left(\sigma_{i^{-}}(f(A))\right)$. Now, $\sigma_{i^{-}}(f(A))$ is $\sigma_{i^{-}}$closed in $Y$ and hence $(i, j)-\omega p(A) \subset$ $f^{-1}\left(\sigma_{i}-(f(A))\right)$, for $(i, j)-\omega p(A)$ is the smallest $(i, j)-\omega$-preclosed set containing $A$. Then $f((i, j)-\omega p(A)) \subset \sigma_{i^{-}}(f(A))$.

(iv) $\Rightarrow$ (iii): Let $F$ be any $\sigma_{i}$-closed subset of $Y$. Then $f\left((i, j)-\omega p\left(f^{-1}(F)\right)\right)$ $\subset \sigma_{i^{-}}\left(f\left(f^{-1}(F)\right)\right) \subset \sigma_{i^{-}}(F)=F$. Therefore, $(i, j)-\omega p\left(f^{-1}(F)\right) \subset f^{-1}(F)$. Consequently, $f^{-1}(F)$ is $(i, j)$ - $\omega$-preclosed in $X$.

(iv) $\Rightarrow(\mathrm{v})$ : Let $B$ be any subset of $Y$. Now, $f\left((i, j)-\omega p\left(f^{-1}(B)\right)\right) \subset \sigma_{i^{-}}$ $\left(f\left(f^{-1}(B)\right)\right) \subset \sigma_{i^{-}}(B)$. Consequently, $(i, j)-\omega p\left(f^{-1}(B)\right) \subset f^{-1}\left(\sigma_{i^{-}}(B)\right)$.

(v) $\Rightarrow$ (iv): Let $B=f(A)$ where $A$ is a subset of $X$. Then, $(i, j)-\omega p(A)$ $\subset(i, j)-\omega p\left(f^{-1}(B)\right) \subset f^{-1}\left(\sigma_{i^{-}}(B)\right)=f^{-1}\left(\sigma_{i^{-}}(f(A))\right)$. This shows that $f((i, j)-\omega p(A)) \subset \sigma_{i^{-}}(f(A))$.

(i) $\Rightarrow$ (vi): Let $C$ be any subset of $Y$. Clearly, $f^{-1}\left(\sigma_{i^{-}}(C)\right.$ is $(i, j)-\omega$-preopen and we have $f^{-1}\left(\sigma_{i^{-}}(C)\right) \subset(i, j)-\omega p\left(f^{-1} \sigma_{i^{-}}(C)\right) \subset(i, j)-\omega p\left(f^{-1}(C)\right)$.

(vi) $\Rightarrow(\mathrm{i})$ : Let $B$ be a $\sigma_{i^{-}}$open set in $Y$. Then $\sigma_{i^{-}}(B)=B$ and $f^{-1}(B) \subset$ 
$f^{-1}\left(\sigma_{i^{-}}(B)\right) \subset(i, j)-\omega p\left(f^{-1}(B)\right)$. Hence we have $f^{-1}(B)=(i, j)-\omega p\left(f^{-1}(B)\right)$. This shows that $f^{-1}(B)$ is $(i, j)-\omega$-preopen in $X$.

Definition 3.4. A collection $\left\{U_{\alpha}: \alpha \in \Delta\right\}$ of $(i, j)$-preopen sets in a bitopological space $\left(X, \tau_{1}, \tau_{2}\right)$ is called an $(i, j)$-preopen cover of a subset $B$ of $X$ if $B \subset \cup\left\{U_{\alpha}: \alpha \in \Delta\right\}$ holds.

Definition 3.5. A bitopological space $\left(X, \tau_{1}, \tau_{2}\right)$ is said to be $(i, j)$-preLindelöf if every $(i, j)$-preopen cover of $X$ has a countable subcover.

A subset $A$ of a bitopological space $X$ is said to be $(i, j)$-preLindelöf relative to $X$ if every cover of $A$ by $(i, j)$-preopen sets of $X$ has a countable subcover.

Theorem 3.6. If $X$ is a bitopological space such that every $(i, j)$-preopen subset is $(i, j)$-preLindelöf relative to $X$, then every subset is $(i, j)$-preLindelöf relative to $X$.

Proof. Let $B$ be an arbitrary subset of $X$ and let $\left\{U_{\alpha}: \alpha \in \Delta\right\}$ be $(i, j)$ preopen cover of $B$. Then the family $\left\{U_{\alpha}: \alpha \in \Delta\right\}$ is an $(i, j)$-preopen cover of the $(i, j)$-preopen set $\cup\left\{U_{\alpha}: \alpha \in \Delta\right\}$. Hence by hypothesis there is a countable subfamily $\left\{U_{\alpha_{i}}: i \in N\right\}$ which covers $\cup\left\{U_{\alpha}: \alpha \in \Delta\right\}$. This subfamily is also a cover of the set $B$.

Theorem 3.7. For any bitopological space $\left(X, \tau_{1}, \tau_{2}\right)$, the following properties are equivalent:

(i) $X$ is $(i, j)$-preLindelöf.

(ii) Every countable cover of $X$ by $(i, j)$ - $\omega$-preopen sets has a countable subcover.

Proof. $\quad(i) \Rightarrow(i i)$ : Let $\left\{U_{\alpha}: \alpha \in \Delta\right\}$ be any cover of $\mathrm{X}$ by $(i, j)$ $\omega$-preopen sets of $X$. For each $x \in X$, there exists $\alpha(x) \in \Delta$ such that $x \in U_{\alpha(x)}$. Since $U_{\alpha}(x)$ is $(i, j)-\omega$-preopen,there exists an $(i, j)$-preopen set $V_{\alpha(x)}$ such that $x \in V_{\alpha(x)}$ and $V_{\alpha(x)} \backslash U_{\alpha(x)}$ is countable. The family $\left\{V_{\alpha(x)}: x \in X\right\}$ is an $(i, j)$-preopen cover of $X$ and $X$ is $(i, j)$-preLindelöf. There exists a countable subset, say $\alpha\left(x_{1}\right), \alpha\left(x_{2}\right), \ldots \alpha\left(x_{n}\right), \ldots \ldots$ such that $X=\cup\left\{V_{\alpha\left(x_{i}\right)}: i \in N\right\}$. Now, we have $\left.X=\bigcup_{i \in N}\left\{V_{\alpha\left(x_{i}\right)} \backslash U_{\alpha\left(x_{i}\right)}\right) \cup U_{\alpha\left(x_{i}\right)}\right\}$ $=\left(\bigcup_{i \in N}\left(V_{\alpha\left(x_{i}\right)} \backslash U_{\alpha\left(x_{i}\right)}\right) \cup\left(\bigcup_{i \in N} U_{\alpha\left(x_{i}\right)}\right)\right)$. For each $\alpha\left(x_{i}\right), V_{\alpha\left(x_{i}\right)} \backslash U_{\alpha\left(x_{i}\right)}$ is a 
countable set and there exists a countable subset $\Delta_{\alpha\left(x_{i}\right)}$ of $\Delta$ such that $V_{\alpha\left(x_{i}\right)} \backslash U_{\alpha\left(x_{i}\right)} \subset \cup\left\{U_{\alpha}: \alpha \in \Delta_{\alpha\left(x_{i}\right)}\right\}$. Therefore, we have $X \subset \bigcup_{i \in N}\left(\cup\left\{U_{\alpha}\right.\right.$ : $\left.\left.\alpha \in \Delta_{\alpha\left(x_{i}\right)}\right\}\right) \cup\left(\bigcup_{i \in N} U_{\alpha\left(x_{i}\right)}\right)$.

$(i i) \Rightarrow(i)$ : Since every $(i, j)$-preopen is $(i, j)-\omega$-preopen, the proof is obvious.

Definition 3.8. A bitopological space $\left(X, \tau_{1}, \tau_{2}\right)$ is called pairwise Lindelöf [1] if each pairwise open cover of $X$ has a countable subcover.

Theorem 3.9. Let $f$ be an $(i, j)$-w-precontinuous function from a space $\left(X, \tau_{1}, \tau_{2}\right)$ onto a space $\left(Y, \sigma_{1}, \sigma_{2}\right)$. If $X$ is $(i, j)$-preLindelöf, then $Y$ is pairwise Lindelöf.

Proof. Let $\left\{V_{\alpha}: \alpha \in \Lambda\right\}$ be a countable cover of $Y$ by $\sigma_{i}$-open sets. Then $\left\{f^{-1}\left(V_{\alpha}\right): \alpha \in \Lambda\right\}$ is an $(i, j)$ - $\omega$-preopen cover of $X$. Since $X$ is $(i, j)$-preLindelöf, there exists a countable subset $\Lambda_{0}$ of $\Lambda$ such that $X=$ $\cup\left\{f^{-1}\left(V_{\alpha}\right): \alpha \in \Lambda_{0}\right\}$; hence $Y=\cup\left\{V_{\alpha}: \alpha \in \Lambda_{0}\right\}$. Therefore $Y$ is pairwise preLindelöf.

Definition 3.10. A function $f:\left(X, \tau_{1}, \tau_{2}\right) \rightarrow\left(Y, \sigma_{1}, \sigma_{2}\right)$ is said to be:

(i) $(i, j)$ - $\omega$-preopen if $f(U)$ is a $(i, j)$ - $\omega$-preopen set of $Y$ for every $\tau_{i}$-open set $U$ of $X$.

(ii) $(i, j)$ - $\omega$-preclosed if $f(U)$ is a $(i, j)$ - $\omega$-preclosed set of $Y$ for every $\tau_{i^{-}}$ closed set $U$ of $X$.

Theorem 3.11. For a function $f:\left(X, \tau_{1}, \tau_{2}\right) \rightarrow\left(Y, \sigma_{1}, \sigma_{2}\right)$, the following statements are equivalent:

(i) $f$ is $(i, j)-\omega$-preopen;

(ii) $f\left(\tau_{i^{-}}(U)\right) \subset(i, j)-\omega p(f(U))$ for each subset $U$ of $X$;

(iii) $\tau_{i}-\left(f^{-1}(V)\right) \subset f^{-1}((i, j)-\omega p(V))$ for each subset $V$ of $Y$. 
Proof. $\quad(i) \Rightarrow(i i)$ : Let $U$ be any subset of $X$. Then $\tau_{i^{-}}(U)$ is a $\tau_{i}$-open set of $X$. Then $f\left(\tau_{i^{-}}(U)\right)$ is a $(i, j)-\omega$-preopen set of $Y$. Since $f\left(\tau_{i^{-}}(U)\right) \subset f(U)$, $f\left(\tau_{i^{-}}(U)\right)=(i, j)-\omega p\left(f\left(\tau_{i^{-}}(U)\right)\right) \subset(i, j)-\omega p(f(U))$.

$($ ii $) \Rightarrow($ iii $)$ : Let $V$ be any subset of $Y$. Then $f^{-1}(V)$ is a subset of $X$. Hence $\left.f\left(\tau_{i^{-}}\left(f^{-1}(V)\right)\right) \subset(i, j)-\omega p\left(f\left(f^{-1}(V)\right)\right) \subset(i, j)-\omega p(V)\right)$. Then $\tau_{i^{-}}$ $\left(f^{-1}(V)\right) \subset f^{-1}\left(f\left(\tau_{i^{-}}\left(f^{-1}(V)\right)\right)\right) \subset f^{-1}((i, j)-\omega p(V))$.

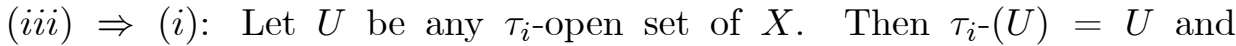
$f(U)$ is a subset of $Y$. Now, $V=\tau_{i^{-}}(V) \subset \tau_{i^{-}}\left(f^{-1}(f(V))\right) \subset f^{-1}((i, j)$ $\omega p(f(V)))$. Then $f(V) \subset f\left(f^{-1}((i, j)-\omega p(f(V)))\right) \subset(i, j)-\omega p(f(V))$ and $(i, j)-\omega p(f(V)) \subset f(V)$. Hence $f(V)$ is an $(i, j)-\omega$-preopen set of $Y$; hence $f$ is $(i, j)-\omega$-preopen.

Theorem 3.12. Let $f:\left(X, \tau_{1}, \tau_{2}\right) \rightarrow\left(Y, \sigma_{1}, \sigma_{2}\right)$ be a function. Then $f$ is an $(i, j)$ - $\omega$-preclosed function if and only if for each subset $V$ of $X,(i, j)$ $\omega p(f(V)) \subset f\left(\tau_{i}-(V)\right)$.

Proof. Let $f$ be an $(i, j)$ - $\omega$-preclosed function and $V$ any subset of $X$. Then $f(V) \subset f\left(\tau_{i^{-}}(V)\right)$ and $f\left(\tau_{i^{-}}(V)\right)$ is an $(i, j)-\omega$-preclosed set of $Y$. We have $(i, j)-\omega p(f(V)) \subset(i, j)-\omega p\left(f\left(\tau_{i^{-}}(V)\right)\right)=f\left(\tau_{i^{-}}(V)\right)$. Conversely, let $V$ be a $\tau_{i}$-closed set of $X$. Then $f(V) \subset(i, j)-\omega p(f(V)) \subset f\left(\tau_{i^{-}}(V)\right)=f(V)$; hence $f(V)$ is an $(i, j)-\omega$-preclosed subset of $Y$. Therefore, $f$ is an $(i, j)-\omega$ preclosed function.

Theorem 3.13. Let $f:\left(X, \tau_{1}, \tau_{2}\right) \rightarrow\left(Y, \sigma_{1}, \sigma_{2}\right)$ be a bijection. Then $f$ is an $(i, j)$ - $\omega$-preclosed function if and only if for each subset $V$ of $Y$, $f^{-1}((i, j)-\omega p(V)) \subset \tau_{i}-\left(f^{-1}(V)\right)$.

Proof. Let $V$ be any subset of $Y$. Then by Theorem 3.12, $(i, j)-\omega p(V) \subset$ $f\left(\tau_{i}-\left(f^{-1}(V)\right)\right)$. Since $f$ is bijection, $f^{-1}((i, j)-\omega p(V))=f^{-1}\left((i, j)-\omega p\left(f\left(f^{-1}(V)\right)\right)\right) \subset$ $f^{-1}\left(f\left(\tau_{i^{-}}\left(f^{-1}(V)\right)\right)\right)=\tau_{i^{-}}\left(f^{-1}(V)\right)$. Conversely, let $U$ be any subset of $X$. Since $f$ is bijection, $(i, j)-\omega p(f(U))=f\left(f^{-1}((i, j)-\omega p(f(U))) \subset f\left(\tau_{i^{-}}\right.\right.$ $\left.\left(f^{-1}(f(U))\right)\right)=f\left(\tau_{i^{-}}(U)\right)$. Therefore, by Theorem 3.12, $f$ is an $(i, j)-\omega-$ preclosed function.

Theorem 3.14. Let $f:\left(X, \tau_{1}, \tau_{2}\right) \rightarrow\left(Y, \sigma_{1}, \sigma_{2}\right)$ be an $(i, j)$ - $\omega$-preopen function. If $V$ is a subset of $Y$ and $U$ is a $\tau_{i}$-closed subset of $X$ containing $f^{-1}(V)$, then there exists an $(i, j)-\omega$-preclosed set $F$ of $Y$ containing $V$ such that $f^{-1}(F) \subset U$. 
Proof. Let $V$ be any subset of $Y$ and $U$ a $\tau_{i}$-closed subset of $X$ containing $f^{-1}(V)$, and let $F=Y \backslash(f(X \backslash U))$. Then $f(X \backslash U) \subset f\left(f^{-1}(Y \backslash V)\right) \subset Y \backslash V$, then $V \subset F$ and $X \backslash U$ is a $\tau_{i}$-open set of $X$. Since $f$ is $(i, j)-\omega$-preopen, $f(X \backslash U)$ is an $(i, j)-\omega$-preopen set of $Y$. Hence $F$ is an $(i, j)-\omega$-preclosed set of $Y$ and $f^{-1}(F)=f^{-1}(Y \backslash(f(X \backslash U)) \subset U$.

Theorem 3.15. Let $f:\left(X, \tau_{1}, \tau_{2}\right) \rightarrow\left(Y, \sigma_{1}, \sigma_{2}\right)$ be an $(i, j)$ - $\omega$-preclosed function. If $V$ is a subset of $Y$ and $U$ is a $\tau_{i}$-open subset of $X$ containing $f^{-1}(V)$, then there exists $(i, j)-\omega$-preopen set $F$ of $Y$ containing $V$ such that $f^{-1}(F) \subset U$.

Proof. The proof is similar to that of Theorem 3.14.

\section{References}

[1] P. Fletcher, H. B. Hoyle, and C. W. Patty, The comparison of topologies, Duke Math. J., 36, pp. 325-331, (1969).

[2] M. Jelic, A decomposition of pairwise continuity, J. Inst. Math. Comput. Sci. Math. Ser., 3, pp. 25-29, (1990).

[3] A. Kar and P. Bhattacharyya, Bitopological preopen sets, precontinuity and preopen mappings, Indian J. Math., 34, pp. 295-309, (1992).

[4] J. C. Kelly, Bitopological spaces, Proc. London Math. Soc., 13, pp. 71-89, (1963).

[5] F. H. Khedr, S. M. Al-Areefi and T. Noiri, Precontinuity and semiprecontinuity in bitopological spaces, Indian J. Pure Appl. Math., 23, pp. 625-633, (1992).

[6] T. Noiri and V. Popa, On weakly precontinuous functions in bitopological spaces, Soochow J. Math., 33(1), pp. 87-100, (2007). 


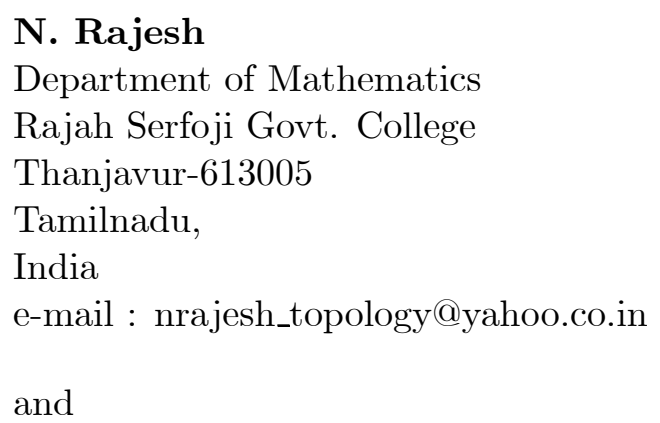

Jamal M. Mustafa

Department of Mathematics

Al al-Bayt University

Mafraq,

Jordan

e-mail : jjmmrr971@yahoo.com 